\title{
1 Evolutionary definition of virtual teams
}

\subsection{INTRODUCTION}

Relatively new literature mentions the concept of virtual teams or virtual management when they emerged around the 1990s. The notion of the concept linked to virtuality and virtual organizations might be interpreted in various forms. In order to clarify the definition of virtual teams, we review the existing academic definitions given to 'virtual teams'. One global definition for 'virtual team' has been suggested, even though there is not yet a widely accepted definition in the research community due to various interpretations of virtuality.

The definitions have evolved during the past two decades, from the initial focus of researchers on virtual teams up to wider consideration integrating today's knowledge and evolution of virtual teaming. Definitions started with gradual approaches to virtuality then began to include a deeper knowledge and understanding of the characteristics, the processes and the challenges arising from virtual teams. Researchers very often compared virtual teams to conventional teams. Then they looked for the differences that existed between virtual teams and co-located teams, and finally they introduced the specific-to-virtual-team dimensions. These dimensions combine the strategy, infrastructure, characteristics and management context of multinationals or any organizations operating in a virtual mode.

They have finally come to the notion of 'virtuality degree' characterizing a team and its level of dispersion which can be temporal, spatial, cultural or organizational. As research progresses, critical factors appear more clearly and can be identified and recognized. Therefore, a more standardized definition can be given.

\subsection{WHAT DOES 'VIRTUAL' MEAN?}

Virtual organizations, virtual teams, virtual work, virtual space ... . A common definition explains that 'virtual' is related to something: 'having 
the essence or the effect but not the appearance or the form of ...' (Dictionary, 2009); and 'not physically existing as such but made by software to appear to do so' (Dictionaries, 2012).

The word 'virtual' takes various meanings when attached to different words. 'Virtual organization' describes disparate conditions for organizations that outsource key components of their production (Robert et al., 1999). Related to teams and according to Jarvenpaa et al. (1998) 'virtual' defines teams created with members issued from different locations to solve problems. They have the appearance of teams but team members are not operating physically in the same location. In parallel, 'virtual work' determinates work done from home, from satellite offices, on the road, or from hotels (Davenport and Pearlson, 1998).

These diverse descriptions refer to one common point: the close relationship of 'virtual' with distance and computer technology (Chudoba et al., 2005). In the case of virtual teams, 'virtual' illustrates the way these teams interact and highlights virtuality as one of the main team characteristics (Griffith and Neale, 2001). Martins and Schilpzand (2011), who analysed the degree of virtuality within global virtual teams, introduce the notion of 'team-ness, virtual-ness and global-ness'. The purpose of these terms is to emphasize the evolution of the 'virtual team' definition around key factors. These factors refer to the challenge in creating an effective team mindset and efficient teamwork, while coping with distance and diversity. They imply the introduction of technology. They also refer to the importance of virtual team processes when defining a virtual team, thus enlarging the focus on conventional team attributes towards virtuality characteristics.

Semantics evolves too and becomes more precise. Virtual could be replaced by 'digital'. However, the term 'digital' is reserved to a system in which the information is recorded or sent out electronically, involving the creation of computer-based representations of physical phenomena (Bailey et al., 2012). Digitization operates as a facilitator over distance and separation between people. 'Digital' is more dedicated to the equipment based on computer technology, such as digital camera or digital boards for instance (Dictionary, 2000, 2012b; Dictionaries, 2012). Whereas, 'virtual' is mainly used for activities or organizations that imitate their real equivalents and occur when physical objects, processes and/or people operate through digitization. 'Digital team' is very rarely used and we found this term almost nonexistent in research literature. Consequently, we keep then the general term 'virtual team' to designate the teams in question.

The designation of virtual activities or organizations is often preceded by the letter 'e' or 'cyber' such as 'e-commerce', 'e-business', 'e-learning', 
'e-team' or 'cyber space'. It points out the link between virtuality and the electronic technology again as these activities cannot be operated without the support of computers, software and the Internet. The evolution of the semantics and the vocabulary are still going on in the field of virtual teams which explains that the expressions are not yet totally concretely designated and may still change in the future according to the proper change of virtual teams and their context.

\subsection{WHAT IS A TEAM?}

In the research papers, the definition of a team very often refers to the one suggested by Katzenbach and Smith (1992). For them, a team is: 'a small group of people with complementary skills who are committed to a common purpose, performance goals and approach for which they hold themselves mutually accountable'. They differentiate the terms 'team' and 'group' because the sense of both terms differs in regards to the way that team and group operate. A group is a collection of individuals gathered for independent tasks that will be consolidated in a final outcome. A group may not constitute a team and does not focus on the coordination of its members at the same level as a team does. The authors point out the key components for an effective team, which include independent or interdependent tasks. A team includes team members, team organization and common objectives. Team members bring their individual competencies such as experience, skills and behaviour in order to contribute and develop the competencies of the whole team. Because they operate with a richness of diverse expertise, virtual teams are the appropriate way to deal with interdependent tasks within a complex context. The organization of the team might differ according to the context, activities, objectives, and management system within which the team functions. Teams can be functional or departmental, cross-functional, and self-managed or led by a manager. No matter the form of organization, the individual and mutual accountability with the team is one of the most critical elements to be considered for a virtual team. Accountability and membership are of great importance in the performance of the team. They also contribute to the synergy of the team and to the coordinated efforts towards common goals. They directly influence the process of a team in regards to the way the team members will work together. 


\subsection{A COMBINED DEFINITION FOR 'VIRTUAL TEAM'}

\subsubsection{Semantic and Evolutionary Context}

Researchers are not all using the same term to identify virtual teams. It seems to depend on the dimension they consider. Either they refer to a group of individuals working together or to a whole entity set up in a more or less complex context. Therefore, various designations appear such as 'virtual group' or 'virtual organization' (Dubinskas and Hargreaves, 1993; Glückler and Schrott, 2007); 'cross-national teams' (Cogburn and Levinson, 2003); 'distributed working team', 'distributed work team' or 'dispersed team', 'geographically distributed teams' (Hinds and Mortensen, 2005); 'remote project teams' (Larbi and Springfield, 2004); 'distributed remote teamwork' (Davidson, 2013); 'cross-cultural virtual team' (Anawati and Craig, 2006; Breitenöder, 2009); 'global virtual team' (Martins and Schilpzand, 2011); 'corporate' or 'global corporate virtual team' (Grosse, 2010); 'far-flung' teams (Watson-Manheim et al., 2012); 'e-teams', 'cyber teams' or 'online teams' (DuFrene and Lehman, 2010); and 'nomadic teams' (Ko et al., 2011). The most frequent name that appears in academic literature is 'global virtual teams' according to Connaughton and Shuffler (2007). The terms frequently associated to virtual teams are 'transnational', 'cross-cultural', 'multicultural', 'intercultural' and 'multinational'. In addition, a rather new expression has emerged to designate the work operated under virtual conditions such as 'virtual teaming'. Among scholars, 'virtuality' or 'virtual-ness' (Martins et al., 2004) has also become a rather common term to describe the virtual context and all the connected characteristics of that context. The definitions of virtual teams have evolved along with the researchers' investigations and their findings which have become more and more focused on the concept. The first definitions mainly addressed the concept of virtual teams in contrast to conventional and co-located or traditional face-toface teams. Team members of co-located teams work in close proximity, geographical, temporal or cultural. Therefore, they easily use informal communication to interact, more specifically for daily interaction and usual visual cues. Most of the time, they are located in the same place, and share common practices and processes. A virtual team is 'an evolutionary form of a network organization' (Miles and Snow, 1986). It is a group of people who can be separated by space, time, and organizations, and work closely together supported by information and communication technologies (ICTs) (Davidow and Malone, 1992; Jarvenpaa and Ives, 1994; Johnson et al., 2001; Martins and Schilpzand, 2011). Mohrman 
(1999) defines virtual teams as 'groups of individuals in different location or business units or companies', 'located in two or more countries' (Martins and Schilpzand, 2011). This definition concerns 'global virtual teams' because virtual team members do not need to be separated by a great distance. They could work in the same building but belong to different divisions of the same corporation and share common activities operated in a virtual team. Usually, this type of virtual team tends to be a cross-functional team, gathering various experts for a common goal. Virtual team members are identified as those 'who share the accountability of a product, service or collective functions or tasks, interdependent in carrying out their accountabilities and thus must work collaboratively to accomplish them' (Mohrman, 1999). Virtual teams are commonly defined as work teams that are 'created, simulated, or carried on by means of a computer or computer network' (Dictionary, 2000). Information and communication technologies are always associated to 'virtual teams'. The community of researchers recognizes that technology is one of the major enablers and drivers of these types of teams. Today, the existence of pure face-to-face teams is rare due to the invasive presence of technology within the business environment. The support of technology aims to increase team productivity, access to information and communication (Arnison and Miller, 2002). 'Virtual teams are teams whose members use technology to varying degrees in working across locational, temporal, and relational boundaries to accomplish an interdependent task' (Martins et al., 2004). The definitions include the idea of a team members' network supported by ICTs for interdependent tasks (Edwards and Wilson, 2004). The definitions then took into account the notion of dispersion, accountability, and collaboration (Dubé and Paré, 2001). Virtual teams are described as an essential element in companies' internal and external environments; dealing with different time zones supported by computermediated communication. As the understanding of virtual teams becomes more precise, critical issues linked to diversity boundaries and interdependent tasks were addressed. It led researchers to identify virtual teams as 'global virtual teams' in the same way the business environment evolves into a global context. In our book, we retain 'virtual team' or 'global virtual team' with no difference, even though global virtual team suggests a broader virtual environment, where team members are working from different international locations, including different management chains and possibly from different organizations. According to this notion, diverse types of formations close to virtual teams have been identified and can be grouped up to organizational virtuality (Hertel et al., 2005; Dubé and Paré, 2001) but remain slightly different from virtual team in regards to the retained definition in our work: 
- Telework or telecommuting teams: members complete their own tasks partially outside the main organization location and work from home most of the time. The terms were introduced in 1975 by Nilles, where telecommunications replace transportation to the office.

- Virtual work groups: different tasks and teleworkers that report to the same manager are combined.

- Virtual teams: members are gathered and interact thanks to technology and ICTs. They share common interests, accountability and goals.

- Virtual communities: larger entities of distributed work where members interact via the Internet, share also common interests and goals, but they are not committed to an organizational structure, and are usually implemented by one of their members.

\subsubsection{Specific Dimensions to Define Virtual Teams}

Some researchers such as Shin (2004) argue that organizations present different levels of virtuality based on four dimensions: temporal, spatial, cultural, and organizational dispersion. O'Leary and Cummings (2007) considered the same dimensions but also introduced, however, the notion of 'degree dispersion' in regards to those four dimensions. The more dispersed the team is, the more complex and virtual it is. They distinguished spatial distance and purely geographical distance with cultural or linguistic distance, known as social distance.

For Kirkman and Mathieu (2005) virtuality indicates the sharing of virtual tools or the dependence on such tools which are needed for the virtual team processes, 'the amount of informational value provided by such tools, and the synchronicity of team member virtual interaction'. Gibson and Gibbs (2006) call it 'electronic dependence' but also add the notion of 'structural dynamism' in reference to frequent changes. Chudoba et al. (2005) widen the mentioned dimension and include work practices as a part of virtual team diversity. As a matter of fact, the work practices may greatly vary within a virtual team, depending on its input characteristics. Chudoba et al. (2005) propose a series of indexes to measure the level of virtuality of a team, based on the concept of discontinuities or changes in expected conditions negatively affecting team cohesion. The discontinuities refer to the dimensions, quoted previously, grouped into team distribution (distance), workplace mobility (dispersion) and the variety of work practices (complexity), partially or totally interrelated. For example, they suggest the following ratios to assess the geographical distance: share of work at home during standard working 
days, share of work while travelling, share of collaboration with people located in various sites, share of collaboration with people who have been met face-to-face. It is relevant to be able to assess or measure the virtuality of the concerned teams because the level of virtuality directly affects the typology of these teams, as well as their processes and consequently, their performance. Chudoba et al. (2005) point out some examples of index used to estimate virtuality. For example, virtuality at a corporate level may be measured according to the proposed training programmes and best practice recommendations in regards to virtual teaming. At the organizational level, the degree of virtuality may be assessed by the investments assumed by an organization in collaboration with the tools to support virtual teaming (computers and various equipment), communication tools (video conferencing, the Internet, and intranet), additional software (group support systems) and training for specific skills. Recent findings take into account three dimensions to assess the virtuality of a team or its virtual distance results, as mentioned by Schweitzer and Duxbury (2010). The first one is the share of time spent by team members working virtually, named 'teamwork virtuality'. Virtual teams whose members work full-time in a virtual mode do not demonstrate the same level of virtuality of those virtual teams whose members work only from time-to-time in such a mode. The second dimension is the share of members within a team who work virtually, in contrast to those who are working co-located. The virtual team management differs if all the virtual team members work in virtual mode as compared to only a few of them working in virtual mode. This dimension is called 'member virtuality' by Schweitzer and Duxbury. The third dimension is the degree of virtual distance between the team members, also known as 'distance virtuality', which includes social distance and possible subdimension such as psychological distance (Wilson et al., 2013).

One additional subdimension could be taken into consideration. The fact that virtual team members frequently belong to several different virtual teams today intensifies the complexity of virtuality, because these virtual team members have to participate in various virtual contexts in a reduced timeframe (Chudoba et al., 2005). The impact of distance on the virtual team boundaries is very different if the virtual team members belong to the same country and culture as compared to being disseminated across continents. The virtual distance comprises the physical, the operational or the daily issues that disturb the virtual team members. It also includes the affinity distance between virtual team members. Affinity distance is the degree to which virtual team members share cultural values, communication style, work practices and social capital (Lojeski and Reilly, 2008). At this level, it is critical to consider that distance and virtuality 


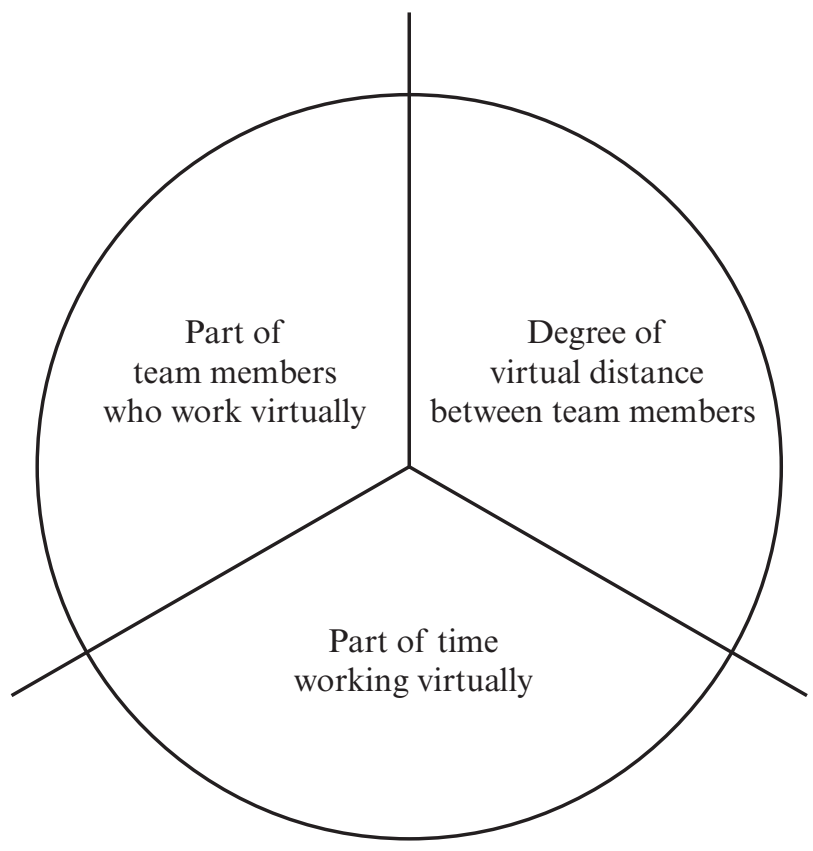

Adapted from Schweitzer and Duxbury (2010)

Figure 1.1 Dimensions that define the virtuality of a work team

alter perception of virtual team members, including oneself (Wilson et al., 2013). Therefore, the dimensions of virtuality no longer focus on the degree of technological means like it did at the emergence of virtual teams. Technology is (or should be) adapted to the virtual team complexity like the degree of virtual team members' cultural dispersion, the duration of the virtual team and the type of interaction, whether synchronous or asynchronous (Jawadi and Boukef Charki, 2011). The levels of complexity introduce the idea of a graduation from a pure traditional face-to-face team to a global virtual team. The further the gradation from a face-to-face team, the more virtual the team is, taking into account that virtual teams are evolving, based on a continuum and that some areas of coexistence of both face-to-face and virtual teams exist (Dixon and Panteli, 2010). It means that research evolves and considers virtual teams on a larger spectrum, which affects the way virtual teamwork might be addressed by organizations. The cursor position shows the possible degree of virtuality for a work team (see Figure 1.2). 


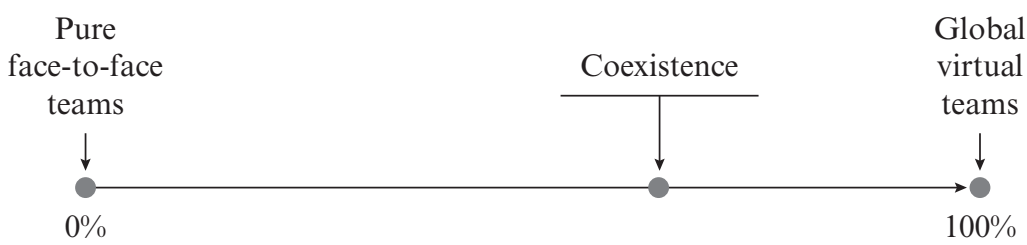

Figure 1.2 Degree of virtuality in virtual teams

\subsection{KEEP IN MIND: THE MAIN ELEMENTS TO DEFINE A VIRTUAL TEAM}

The definition of the terms 'virtual' and 'team' contributes to the understanding of what a virtual team is. Virtual teams gather members separated by one or more forms of distance thus influencing behaviours and characteristics that alter the group processes (Wilson et al., 2013).

- The key points to be addressed in the virtual team definition are virtual distance which includes time, space and organizational boundaries, global dispersion, degree of virtuality and webs of technology to collaborate and communicate. These critical elements shape the complexity of a virtual team and affect its functioning and performance.

- A virtual team is determinate by its degree of virtuality. Virtuality level depends on the share of time spent for virtual work, the share of team members working virtually, and the degree of virtual distance between the team members.

- The degree of virtuality affects the complexity of the virtual team and, therefore, its effectiveness. The effectiveness of a virtual team directly depends on the capacity of the individuals within the virtual team to work together toward common goals, to develop and reach performance outcomes, and to remain mutually accountable. Collaboration and coordination appear to be essential in the definition of virtual team, especially because the degree of dispersion is high and so is reliance on technologies.

The above points illustrate the reason why the definition of a virtual team evolves with time, according to the global context. Therefore, a virtual team can no longer be definitively defined in contrast to a face-to-face team. 


\subsection{CONCLUSION}

The dichotomy between a face-to-face and a virtual team is no longer mentioned as often as it used to be in the early phase of research on virtual teams. To date, the definition of 'virtual team' as based on semantics is more explicit. We could not find any widely accepted definition of virtual teams within the academic research due to the evolution of virtual teaming. The researchers' conclusions admit that the definition goes beyond semantics and involves more elements and dimensions, such as virtuality. The assessment of virtuality in relation to virtual team or virtual teaming seems vague and difficult to evaluate and measure. However, virtuality is one of the most efficient ways to indicate the specific characteristics of virtual teams. Virtuality creates the complexity of virtual teams and has a great implication on their operating processes and outcomes. The topic is gaining prominence in academic literature.

Based on current research results, the notion and concept evoked in the global definition of virtual teams contribute determining the typology that characterizes a virtual team. Inputs, processes and outputs of virtual teams are detailed in this book to assist in understanding how virtual teams operate. We link them to the involved dimensions that affect the typology of a virtual team, the work practices, the organization and the technology. The next chapter focuses on inputs that are essential to identity and differentiate virtual teams, especially for individuals directly involved in virtual teams either as a leader or as a member.

\subsection{GUIDELINES FOR MANAGERIAL PRACTICE: ASSESS THE VIRTUALITY OF YOUR VIRTUAL TEAM}

One critical factor for a virtual team manager is to estimate the level of complexity and virtuality of the team to be managed. In virtual team management, complexity is defined by all elements that connect the team with dispersion along with the team composition and its processes within an organization. The share of virtual processes in the team indicates the level of virtuality, which partly increases or decreases the level of complexity of the team. Therefore, the way the team can be managed is directly impacted. The virtual team management also depends on the type of technological support dedicated to the concerned team, which is important to list in order to figure out the situation. A clear situation enables the manager to complete the necessary technical equipment when the support does not 
Table 1.1 Assess the virtuality of your virtual team

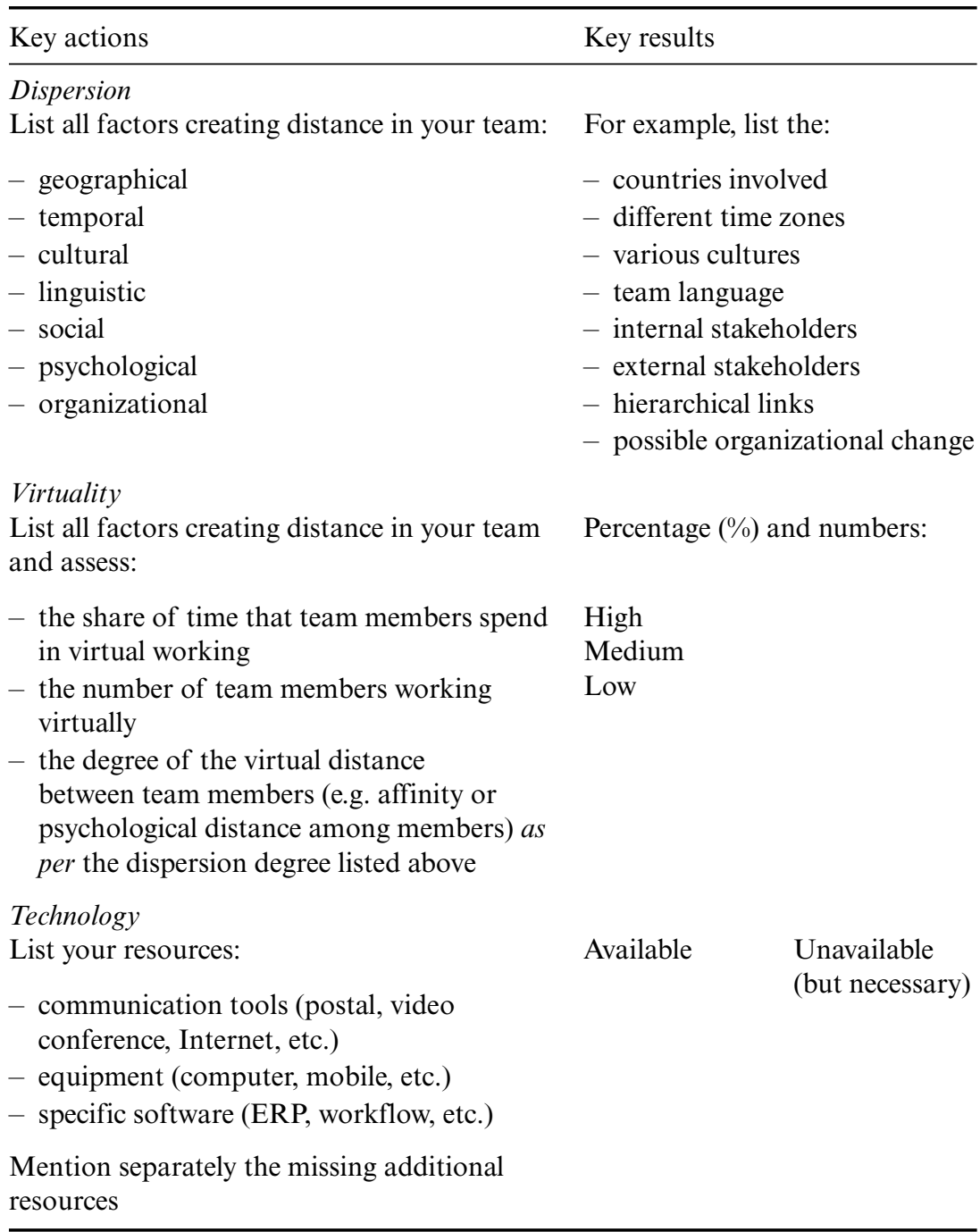

match the virtual characteristics of the team. The guidelines invite the virtual team manager to identify the level of complexity and virtuality of the team. 